\title{
Osteoartrite de joelho e o aspirado de medula óssea como escolha de tratamento -
}

\section{Uma revisão narrativa}

\author{
Knee osteoarthritis and bone marrow aspirate as a treatment choice - Narrative review \\ La Osteoartritis de rodilla y el aspirado de médula ósea como opción de tratamiento: Una revisión
}

narrativa

Recebido: 22/05/2021 | Revisado: 29/05/2021 | Aceito: 02/06/2021 | Publicado: 18/06/2021

\author{
Paulo Fernando Andrade Cunha \\ ORCID: https://orcid.org/0000-0003-4728-9051 \\ Universidade Tiradentes, Aracaju, Sergipe, Brasil \\ E-mail: andradecunha.paulo@hotmail.com \\ Ronald Bispo Barreto da Silva \\ ORCID: https://orcid.org/0000-0002-8500-1904 \\ Universidade Tiradentes, Aracaju, Sergipe, Brasil \\ E-mail: dr.ronaldbarreto@gmail.com
}

\begin{abstract}
Resumo
Introdução: Osteoartrite degenerativa (OA) é a principal causa de dor e incapacidade física no mundo, além de estar relacionada com mais de 100 condições reumatológicas e ortopédicas que acometem as articulações e seus tecidos. Objetivo: Compilar, em português, informações sobre o uso do aspirado de medula óssea no tratamento da OA de joelho. Metodologia: Revisão narrativa realizada através de fontes literárias e cientificas, por meio da plataforma US National Library of Medicine (PUBMED). Os Descritores em Ciência da Saúde utilizados foram: knee osteoarthritirs, epidemiology, etiology, pathophysiology, treatment, bone marrow aspirate. Discussão: OA de joelho tem mecanismos que causam inflamação em algum grau nos condrócitos e nas células sinoviais, liberando algumas citocinas que diminuem a síntese de colágeno, além de estimular mediadores catabólicos, causando apoptose dos condrócitos. Assim, o aspirado de medula óssea (BMA) surge como uma terapia celular inovadora, já que as suas células-tronco mesenquimais (MSC) tem um grande potencial imunomodulador, apesar de seus mecanismos ainda não serem completamente compreendidos. Conclusão: novas opções terapêuticas, como as MSC obtidas por BMA, surgem para suprir os espaços deixados pelos tratamentos mais usados atualmente, devido a sua interação imunomoduladora. De certo que existem ainda lacunas sobre seus mecanismos, sendo necessários mais estudos acerca do tema, a fim de consolidá-lo como um tratamento totalmente viável para OA, como pode-se concluir dos estudos promissores existentes.
\end{abstract}

Palavras-chave: Osteoartrite do joelho; Células-tronco mesenquimais da medula óssea; Medicina regenerativa.

\begin{abstract}
Introduction: Osteoarthritis (OA) is the leading cause of pain and physical disability in the world, in addition to being related to more than 100 rheumatological and orthopedic conditions that affect the joints and their tissues. Objective: Compile, in portuguese, information on the use of boné marrow aspirate in the treatmen of knee OA. Methods: Narrative review conducted through literary and scientific sources, through the platform US National Library of Medicine (PUBMED). The Health Science Descriptors used were: knee osteoarthritis, epidemiology, etiology, pathophysiology, treatment, bone marrow aspirate. Discussion: OA has mechanisms that cause inflammation to some degree in chondrocytes and synovial cells, releasing some cytokines, that decrease collagen synthesis and stimulate catabolic madiators, causing apoptosis to chondrocytes. Thus, bone marrow aspirate (BMA) emerges as an interesting cell therapy, since its mesenchymal stem cells (MSC), has a great immunomodulatory potential, although its mechanisms are not yet fully understood. Conclusion: new therapeutic options, such as MSC obtained by BMA, appear to supply the spaces left by the most used treatments today, due to their immunomodulatory interaction. Certainly, there are still gaps in its mechanisms, requiring more studies on the topic, in order to consolidate it as a totally viable treatment for OA, as can be conclused from the promising existing studies.
\end{abstract}

Keywords: Osteoarthritis of the knee; Bone marrow mesenchymal stem cells; Regenerative Medicine.

\section{Resumen}

Introducción: La osteoartritis degenerativa $(\mathrm{OA})$ es la principal causa de dolor físico y discapacidad en el mundo, además de estar relacionada con más de 100 afecciones reumatológicas y ortopédicas que afectan las articulaciones y sus tejidos. Objetivo: recopilar información en portugués sobre el uso del aspirado de médula ósea en el tratamiento de la artrosis de rodilla. Metodología: Revisión narrativa realizada a través de fuentes literarias y científicas, a través de la plataforma US National Library of Medicine (PUBMED). Los descriptores de ciencias de la salud utilizados 
fueron: artrosis de rodilla, epidemiología, etiología, fisiopatología, tratamiento, aspirado de médula ósea. Discusión: La OA de rodilla tiene mecanismos que provocan inflamación en algún grado en condrocitos y células sinoviales, liberando algunas citocinas que disminuyen la síntesis de colágeno, además de estimular mediadores catabólicos, provocando la apoptosis de condrocitos. Así, el aspirado de médula ósea (BMA) aparece como una terapia celular innovadora, ya que sus células madre mesenquimales (MSC) tienen un gran potencial inmunomodulador, aunque sus mecanismos aún no se conocen del todo. Conclusión: nuevas opciones terapéuticas, como las MSC obtenidas por BMA, parecen complementar los espacios que necesitan los tratamientos más mal utilizados en la actualidad, a través de su interacción inmunomoduladora. Ciertamente, existen vacíos en sus mecanismos, requiriendo malos estudios sobre el tema, con el propósito de consolidarlo como un tratamiento totalmente viable para la OA, como promete desde los prometedores estudios existentes.

Palabras clave: Artrosis de rodilla; Células madre mesenquimales de la médula ósea; Medicina regenerativa.

\section{Introdução}

Osteoartrite degenerativa é a principal causa de dor e incapacidade física no mundo (Chu; Millis; Olson, 2014), além de estar relacionada com mais de 100 condições reumatológicas e ortopédicas que acometem as articulações e seus tecidos (Lespasio, 2017). Tratando-se especificamente do joelho, articulação mais acometida pela OA nos Estados Unidos (Lawrence et al., 2008), a incidência desta apenas tende a aumentar com o passar dos anos, devido ao crescimento da obesidade na sociedade (Lespasio, 2017).

A artrose de joelho acomete cerca de 33,6\% dos americanos com mais de 65 anos (Lawrence et al., 2008), sendo mais frequente e sintomático nas mulheres (Roos; Arden, 2016). Além da idade avançada, sexo feminino e obesidade, também são fatores de risco para OA no joelho: o sobrepeso, lesões prévias de joelho, artrose de mão/presença de nódulos de Herberden, tabagismo, trabalhos que exigem ajoelhar e levantar com frequência, atividade física intensa e algumas comorbidades, como cardiopatias e depressão (Silverwood et al., 2015). Portanto, todos esses fatores têm grande influência no joelho por causarem inflamação em algum grau nos condrócitos e nas células sinoviais, liberando citocinas como a interleucina $1 \beta$ (IL-1 $\beta$ ) e o fator de necrose tumoral alfa (TNF- $\alpha$ ), que vão diminuir a síntese de colágeno, além de estimular mediadores catabólicos, tais como: metaloproteinases, interleucina 8 (IL-8), interleucina 6 (IL-6), prostaglandina E2 (PGE2) e óxido nítrico (NO). Dessa forma, o conjunto do processo inflamatório com o estresse mecânico vai causar apoptose e catabolismo dos condrócitos, o que gera a OA (Rezende; Campos; Pailo, 2013) (Rezende et al., 2011).

Sendo assim, em 2009, a Sociedade Internacional de Pesquisa em Osteoartrite (Osteoarthritis Research Society International - OARSI) publicou um guia de recomendação de condutas para o tratamento da OA, o qual se baseia numa combinação de medidas farmacológicas e não-farmacológicas. Dentre elas estão a perda de peso, prática regular de exercícios físicos adequados, fisioterapia, acupuntura, analgésicos orais, condroprotetores orais e injetáveis, infiltrações com corticoide e procedimentos cirúrgicos. (Zhang et al., 2010). Entretanto, esses métodos podem ser: meramente sintomáticos, como as infiltrações de corticosteroides; ou muito invasivos, no caso da artroplastia, com chances de complicações (morte, embolia pulmonar, acidente vascular cerebral e infarto do miocárdio).

$\mathrm{O}$ aspirado de medula óssea surge como terapia celular inovadora, já que sua técnica é simples, tem baixa morbidade e fornece células-tronco mesenquimais. Estas vão atuar no reparo tecidual da articulação, além de influenciar na IL-8 e IL-1 $\beta$ e servir como fonte de peptídeos de sinalização intracelular, dentre eles: fator de crescimento derivado de plaquetas (PDGF), fator de crescimento transformador-beta (TGF- $\beta$ ) e fator de crescimento endotelial vascular (VEGF) (Piuzzi et al., 2018).

Com isso, esse artigo tem por objetivo compilar, em português, informações sobre o uso do aspirado de medula óssea no tratamento da $\mathrm{OA}$ de joelho. Pretendendo que mais pessoas se interessem e pesquisem temas relacionados à Medicina regenerativa, sendo esta possível e confiável método de tratamento. 


\section{Metodologia}

Trata-se de uma revisão narrativa que visa melhorar o conhecimento sobre a OA de joelho e o BMA como uma vertente de tratamento.

Este estudo foi realizado através de fontes literárias e científicas. A coleta de dados foi realizada através da plataforma US National Library of Medicine (PUBMED). Os Descritores em Ciência da Saúde utilizados para a busca foram: knee osteoarthritis, epidemiology, etiology, pathophysiology, treatment, bone marrow aspirate. Os critérios de inclusão para os artigos selecionados pelo mecanismo de busca foram: ensaios clínicos randomizados e não randomizados; revisões narrativas e revisões sistemáticas, nos idiomas inglês e português, com recorte temporal entre os anos de 2001 a 2021 , que se adequaram ao propósito da pesquisa (com prioridade aos artigos publicados nos últimos 5 anos). Foram excluídos os artigos que não estavam atrelados à finalidade da pesquisa, por meio da leitura do título e do resumo. Além disso, as referências dos artigos que foram escolhidos, e que atendiam as necessidades da pesquisa, também foram analisadas. A busca bibliográfica foi realizada durante os meses de abril de 2021 e maio de 2021. Diante do processo de seleção dos artigos foram aplicadas as etapas de identificação, triagem e elegibilidade, tendo em vista a leitura na íntegra dos artigos selecionados. Inicialmente, foram encontrados 95 artigos no total. Após a seleção feita a partir dos critérios de inclusão, restaram 49 artigos. Por fim, foi realizada a avaliação crítica dos estudos elegidos para posterior dissertação e discussão da temática para disseminação do conhecimento obtido.

\section{Discussão}

\subsection{Fisiologia do joelho e fatores desencadeantes da $\mathrm{OA}$}

O joelho é composto anatomicamente e histologicamente por: cartilagem articular, osso subcondral, sinóvia, coxim adiposo infrapatelar, patela, meniscos, músculos, ligamentos e tendões (Flandry; Hommel, 2011).

A cartilagem articular do joelho é uma estrutura avascular, sem tecidos linfáticos ou nervosos (Martel-Pelletier et al., 2016). Ela é constituída por condrócitos e matriz extracelular, esta composta por água (70\%), colágeno (tipos 2, 3, 6, 9 e 11), agrecanos, glicosaminoglicanos e glicoproteínas (Xia et al., 2014). Esses componentes se organizam por ligações de cargas negativas, junto ao ácido hialurônico, formando uma estrutura organizada e resistente (Goldring; Goldring, 2016). Já os condrócitos são as principais unidades funcionais das articulações, conseguindo sintetizar todos os componentes da matriz extracelular, mantendo a homeostase do tecido (Goldring; Marcu, 2009).

Os condrócitos são regulados por mecanorreceptores de superfície. Esses nada mais são do que canais iônicos e integrinas sensíveis a estímulos mecânicos. A estimulação desses receptores inicia uma cascata de reações que leva ao processo de remodelamento da cartilagem. Impactos de exercícios moderados reduzem a síntese de enzimas proteolíticas, prevenindo a progressão de lesões. Já a falta de estimulação, como em um paciente imobilizado, pode deixar a cartilagem menos espessa ou amolecida, quando comparada a um paciente normal. Ao contrário de um estímulo excessivo que pode desencadear reações catabólicas na articulação, fator também desencadeante para OA (Musumeci, 2016).

O osso subcondral tem íntima interação com a cartilagem articular, tanto para homeostase como para acelerar o desgaste da OA, sendo composto por duas estruturas: osso cortical, mais externamente; e osso trabecular, mais interno (FunckBrentano; Cohen-Solal, 2015). Suas principais células são os osteoblastos e osteoclastos, responsáveis pela síntese e remodelagem dos ossos. Essas células também respondem aos estímulos mecânicos de forma similar aos condrócitos, liberando citocinas pró-inflamatórias e enzimas degenerativas (Sanchez et al., 2012). Assim, num estado de OA instalada, o osso cortical fica mais volumoso e espesso, enquanto o osso trabecular fica deteriorado, podendo até esclerosar, formando alterações ósseas, como cistos e osteófitos (Goldring, 2009). 
A sinóvia é formada por um conjunto de sinoviócitos, que, dentre outros elementos, compõem a membrana sinovial, bem como pelo fluido sinovial. Este funciona como fonte de nutrientes para a cartilagem articular, já que esta é avascular, e como reservatório para material degradado (Flandry; Hommel, 2011). Já a membrana sinovial tem duas camadas: íntima e subíntima. Juntas tem cinco milímetros de espessura em pessoas sadias. Por sua vez, os sinoviócitos são as principais células para o metabolismo sinovial, onde também se encontram os macrófagos que, quando estimulados, produzem VEGF, mecanismo estimulador para desenvolvimento inflamatório da OA, causando sinovite (Belluzzi et al., 2019). Durante essa inflamação são liberados TNF- $\alpha$ e IL-1 $\beta$, recrutando mastócitos e linfócitos T (principalmente Th1) e B, além dos macrófagos da sinóvia. Somados, este infiltrado inflamatório produz IL-6, IL-8, IL-15, IL-17 e prostaglandina E2 (PGE2), liberando enzimas proteolíticas que causam degradação da cartilagem articular, cujo seu catabolismo estimula retroativamente a sinovite (Belluzzi et al., 2019) (Sellam; Berenbaum, 2010).

Compõem as partes moles do joelho o coxim adiposo infrapatelar, meniscos, musculatura periarticular, ligamentos e tendões, cada um, quando lesionado, colaborando de alguma forma para o surgimento da OA. O coxim é inervado por fibras do tipo-C, estimuladas nos pacientes com OA, emitindo a sensação de dor causada pela isquemia e necrose desse tecido adiposo, por conta do infiltrado inflamatório (Belluzzi et al., 2019). Já a lesão dos meniscos é um importante fator de risco, pois leva a uma instabilidade de toda articulação, além de problemas de lubrificação da face articular, perda de amortecimento de choque e suscetibilidade a altas cargas, predispondo a danos na cartilagem articular e osso subcondral, e, consequentemente, a OA de joelho (Englund et al., 2012). Além disso, a fraqueza dos músculos periaritulares (quadríceps e isquiotibiais) é fator de risco para o desenvolvimento da OA (Kim; Yoo; Kim, 2018). Outrossim, a musculatura de pacientes acometidos libera miocinas, que interage com as outras estruturas do joelho, facilita o desenvolvimento da OA (Krishnasamy; Hall; Robbins, 2018). Por fim, $50 \%$ dos pacientes que lesionam tendões ou ligamentos, principalmente o cruzado anterior do joelho, dos 10 20 anos, gerando instabilidade, tem predisposição para desenvolver OA no futuro (Wu et al., 2020).

\subsection{Mecanismos inflamatórios da $\mathrm{OA}$}

A OA tem influência mecânica, além de grande atuação inflamatória, gerando sintomas de dor, inchaço e rigidez (Goldring; Goldring, 2007). Existe um balanço entre citocinas pró-inflamatórias e anti-inflamatórias que servem como mediadores para o equilíbrio metabólico do joelho. Dentre as pró-inflamatórias estão TNF- $\alpha$, IL-1 $\beta$, IL-6, IL-8 e IL-17 e as anti-inflamatórias seriam IL-1Ra, IL-4, IL-10 e IL-13 (Mabey et al., 2016). O TNF- $\alpha$ e IL-1 $\beta$ induzem produção de PGE2 e algumas desintegrinas conhecidas como ADAMTS, além de ativar alguns genes, dentre eles o NF-kB, sendo este o principal mediador catabólico dos condrócitos. O NF-kB expressa a liberação de HIF2 $\alpha$, estimulando a degradação da matriz extracelular e apoptose dos condrócitos (Yang et al., 2019). Outros fatores inflamatórios induzidos por este gene seriam óxido nítrico sintase (NOS2), COX-2 e IL-1, sendo esta interleucina a estimuladora da cascata inflamatória de forma cíclica, induzindo a produção de IL-6, IL-8, IL-17 e IL-18. Os ADAMTS também estimulam a produção de IL-1(Goldring; Goldring, 2007) (Loeser et al., 2012).

\subsection{Tratamento e a Medicina regenerativa}

Com os conhecimentos acerca da inflamação na OA, o BMA surge como uma opção terapêutica (Piuzzi et al., 2018). Sua base funcional são as células-tronco mesenquimais, pluripotentes, isto é, habilidade de se diferenciar em diversos tecidos, como osteócitos, condrócitos, adipócitos, mastócitos, fibroblastos e células precursoras da hematopoiese (Gobbi et al., 2017). Além disso, possuem capacidades imunomoduladoras e supressivas para apoptose dos condrócitos (Hong; Traktuev; March, 2010). 


\subsubsection{As células-tronco mesenquimais}

As MSC são células precursoras com alto potencial de diferenciação e multiplicação, podendo substituir células lesadas, como os condrócitos na OA (Caplan, 2019). Existem diferentes fontes para se coletar MSC. As duas autólogas utilizadas são da medula óssea e do tecido adiposo (Prodromos et al., 2020). Seus mecanismos fisiológicos ainda estão em estudo, mas as MSC possuem 3 meios já conhecidos que agem no tratamento da AO, são eles: a expressão de MicroRNA (MiRNA), conseguindo regular a tradução de mRNA de células vizinhas, que podem estimular o ambiente ao seu favor; a estimulação do reparo natural dos condrócitos pela secreção de fatores angiogênicos (VEGF e TGFB1), inibidores de apoptose (STC-1, SFRP2, TGFB1 e HGF), mitóticos (TGF-a, TGF-B, HGF, IGF-1, FGF-2, EGF) e anti-fibrose (KGF, SDF1, MIP1a, MIP1b); a imunomodulação, suprimindo as células Th1 e estimulando as Th2, trocando ainda os tipos de macrófagos e inibindo os linfócitos B (Mancuso et al., 2019) (Pittenger et al., 2019) (Ren et al., 2008).

\subsubsection{BMA como opção de tratamento}

Uma das vantagens da BMA como fonte de MSC é que sua técnica é simples. Trata-se de um procedimento percutâneo, com baixa morbidade, por ser fonte autóloga, utilizando-se apenas de uma suspensão de única célula que pode ser facilmente processada, diferente de fontes adiposas que precisam de várias etapas de processamento. (Piuzzi et al., 2018) (Jäger et al., 2010).

O local de escolha para obter o aspirado geralmente é a crista ilíaca posterior, local seguro, que apresenta menos complicações e tem uma maior quantidade de MSC, se comparada com a crista ilíaca anterior (Pierini et al., 2013). Caso a pessoa tenha alguma contraindicação à coleta posterior, pode ser feito o aspirado na região anterior (Piuzzi et al., 2018). O principal fator da técnica é manter a pressão de aspiração constante e em baixos níveis, escolhendo seringas de $10 \mathrm{ml}$ (Hernigou et al., 2013). Deve ser assim por que as MSC estão diluídas em sangue quando aspiradas, fazendo com que $85 \%$ das células disponíveis sejam coletadas nos primeiros $2 \mathrm{ml}$ de aspirado. Dessa forma, a cada $2 \mathrm{ml}$ coletados, a agulha deve avançar 0,5 a $1 \mathrm{~cm}$ para otimizar a coleta (Piuzzi et al., 2018). Entretanto, ainda não existe consenso sobre alguns aspectos da técnica, como posição do paciente, anestesia e escolha das agulhas de coleta (Kim et al., 2020) (Everts et al.). No mais, ainda pode ser feita uma variação desse método através da centrifugação do aspirado coletado, separando por densidade as MSC do plasma e células sanguíneas, formando o concentrado de aspirado de medula óssea (BMAC) (Hegde et al., 2014).

Se por um lado não há consenso entre alguns fatores da técnica, por outro, estudos já mostram a efetividade do BMA como tratamento para AO como método seguro.

Um estudo prospectivo de 5 anos, feito com 60 pacientes, com idade média de 44 anos, escolhidos randomicamente, fez a coleta na crista ilíaca (sem preferência entre porção anterior ou posterior), aplicou BMAC via artroscopia, e avaliou esses participantes em diversos intervalos durante o período de pesquisa, utilizando escores funcionais e ressonâncias magnéticas para verificar a evolução de cada um. Ficou evidenciado um pico de melhora nos primeiros seis meses, com leve evolução até dois anos de acompanhamento, e estabilizando, sem muita melhora, até o quinto ano de estudo (Shetty et al., 2018).

Outra evidência da sua efetividade é uma pesquisa publicado com 18 pacientes maiores de 35 anos, utilizando-se da punção da crista ilíaca posterior, aplicando o BMA de forma intra-articular, guiado por referências anatômicas. Esses pacientes foram avaliados com um questionário de funcionalidade e qualidade de vida, chamado Knee and Osteoarthritis Outcome Score (KOOS), nos intervalos de 1, 2, 3, 6, 9 e 12 meses. Semelhante ao outro estudo, foi evidenciado um pico de melhora inicial nos dois primeiros meses, estabilizando até o nono mês e voltando a ter uma leve melhora no décimo segundo mês (Bastos et al., 2018).

Mais um artigo publicado, sobre 15 pacientes portadores de OA severa acompanhados por dois anos, mostrou a efetividade do BMAC. Os participantes tinham idade média de 48 anos e precisavam ter IMC $<30 \mathrm{~kg} / \mathrm{m}^{2}$. Tais participantes 
passaram por avaliações radiológicas e diversos questionários sobre funcionalidade e qualidade de vida, como KOOS, SF-36 health score e Lysholm. O local de coleta do BMA foi a crista ilíaca aplicada via artroscópica. Esses pacientes foram reavaliados com 6, 12 e 24 meses. Todas análises apresentaram melhora expressiva dos pacientes durante todo tempo de pesquisa (Gobbi et al., 2011).

Um outro estudo com 41 pacientes durante doze meses, dessa vez com qualquer grau de AO, também mostrou resultados animadores. A idade média deles era de 60 anos. O BMAC foi coletado da crista ilíaca e aplicado por artroscopia. Os participantes foram submetidos a escores funcionais e questionados quanto a sua sintomatologia. Um fator interessante sobre essa pesquisa é que os pacientes foram proibidos de utilizar AINEs durante todo período de estudo e de praticar exercícios intensos nas primeiras seis semanas, com intuito de evitar interferências. Eles foram avaliados no pré-operatório, no pós-operatório imediato, com 3, 6 e 12 meses, após aplicação. Nos três primeiros meses houve o pico da melhora de todos os parâmetros analisados, mantendo uma leve melhora até o fim do acompanhamento (Kim et al., 2014).

Outra pesquisa utilizando controle, separou 61 participantes em 2 grupos: 30 no grupo de estudo (recebendo aplicação de BMA retirado da crista ilíaca posterior e aplicado via intra-articular) e 31 no grupo controle (recebendo solução salina, como placebo). Esses pacientes tinham que ter no mínimo 30 anos e OA grau 2 ou 3 de Kellgren-Lawrence. Eles foram acompanhados por seis meses, sendo avaliados antes da aplicação e após uma semana, um mês e seis meses. Os parâmetros de avaliação foram o questionário WOMAC e escala visual analógica (VAS). Aqui os pacientes apresentaram grande avanço, principalmente na VAS, sendo superior ao grupo controle, que não teve grandes alterações (Garay-Mendoza et al., 2018).

Entretanto, outro estudo que acompanhou por seis meses 25 pacientes portadores de OA bilateral dos joelhos, tiveram ambos analisados. Em um dos joelhos foi aplicado BMAC retirado da crista ilíaca (grupo de estudo) e no outro um placebo com solução salina (grupo controle). Todos os participantes foram analisados com escores funcionais, escala visual da dor, algometria, ressonância magnética e coleta do líquido sinovial. Como resultado foi apresentado que os joelhos tratados com BMAC tiveram uma melhora até o terceiro mês, estabilizando-se até o sexto mês. Contudo, os joelhos tratados com placebo também obtiveram valores parecidos, mostrando que não houve grandes diferenças entre o grupo de estudo e o de controle, evidenciando que ainda há o que se esclarecer sobre o mecanismo das células-tronco mesenquimais (SHAPIRO et al., 2017).

Ressalte-se que todos esses artigos relataram que não houve efeitos adversos significativos nos pacientes que participaram das pesquisas, salvo os mais comuns detectados como: derrame articular, calor na articulação, eritema articular e alteração da amplitude do joelho (Shapiro et al., 2019).

\section{Conclusão}

Diante de tudo o que foi apresentado e estudado, a OA é um acometimento articular com fatores mecânicos que desencadeiam reações de caráter inflamatório para a sua fisiopatologia. Em combate, novas opções terapêuticas, como as MSC obtidas por BMA, surgem para suprir os espaços deixados pelos tratamentos mais usados atualmente, devido a sua interação imunomoduladora. De certo que existem ainda lacunas sobre seus mecanismos, sendo necessários mais estudos futuros acerca do tema, principalmente com grupo controle e maior número de participantes, a fim de consolidá-lo como um tratamento totalmente viável para OA, como pode-se concluir dos estudos promissores existentes.

\section{Referências}

Bastos, R., Mathias, M., Andrade, R., Bastos, R., Balduino, A., Schott, V., \& Espregueira-Mendes, J. (2018). Intra-articular injections of expanded mesenchymal stem cells with and without addition of platelet-rich plasma are safe and effective for knee osteoarthritis. Knee Surgery, Sports Traumatology, Arthroscopy, 26(11), 3342-3350.

Belluzzi, E., Stocco, E., Pozzuoli, A., Granzotto, M., Porzionato, A., Vettor, R., \& Macchi, V. (2019). Contribution of infrapatellar fat pad and synovial membrane to knee osteoarthritis pain. BioMed research international, 2019. 
Caplan, A. I. (2019). Medicinal signalling cells: they work, so use them. Nature, 566(7742), 39-40.

Chahal, J., Gómez-Aristizábal, A., Shestopaloff, K., Bhatt, S., Chaboureau, A., Fazio, A., \& Viswanathan, S. (2019). Bone marrow mesenchymal stromal cell treatment in patients with osteoarthritis results in overall improvement in pain and symptoms and reduces synovial inflammation. Stem cells translational medicine, 8(8), 746-757.

Centeno, C., Pitts, J., Al-Sayegh, H., \& Freeman, M. (2014). Efficacy of autologous bone marrow concentrate for knee osteoarthritis with and without adipose graft. BioMed research international, 2014.

Chu, C. R., Millis, M. B., \& Olson, S. A. (2014). Osteoarthritis: from palliation to prevention: AOA critical issues. The Journal of bone and joint surgery. American volume, 96(15).

Englund, M., Roemer, F. W., Hayashi, D., Crema, M. D., \& Guermazi, A. (2012). Meniscus pathology, osteoarthritis and the treatment controversy. Nature Reviews Rheumatology, 8(7), 412

Everts, P. A., Ferrell, J., Mahoney, C. B., II, G. F., Irizarry-de Roman, M., Paul, R., \& Mautner, K. (2020). A Comparative Quantification in Cellularity of Bone Marrow Aspirated with two New Harvesting Devices, and The Non-equivalent Difference Between A Centrifugated Bone Marrow Concentrate And A Bone Marrow Aspirate As Biological Injectates, Using A Bi-Lateral Patient Model.

Flandry, F., \& Hommel, G. (2011). Normal anatomy and biomechanics of the knee. Sports medicine and arthroscopy review, 19(2), 82-92.

Funck-Brentano, T., \& Cohen-Solal, M. (2015). Subchondral bone and osteoarthritis. Current opinion in rheumatology, 27(4), 420-426.

Garay-Mendoza, D., Villarreal-Martínez, L., Garza-Bedolla, A., Pérez-Garza, D. M., Acosta-Olivo, C., Vilchez-Cavazos, F., \& Mancías-Guerra, C. (2018). The effect of intra-articular injection of autologous bone marrow stem cells on pain and knee function in patients with osteoarthritis. International journal of rheumatic diseases, 21(1), 140-147.

Gobbi, A., Espregueira-Mendes, J., Lane, J. G., \& Karahan, M. (2017). Bio-orthopaedics. Berlin, Heidelberg: Springer Berlin Heidelberg.

Gobbi, A., Karnatzikos, G., Scotti, C., Mahajan, V., Mazzucco, L., \& Grigolo, B. (2011). One-step cartilage repair with bone marrow aspirate concentrated cells and collagen matrix in full-thickness knee cartilage lesions: results at 2-year follow-up. Cartilage, 2(3), 286-299.

Goldring, M. B., \& Marcu, K. B. (2009). Cartilage homeostasis in health and rheumatic diseases. Arthritis research \& therapy, 11(3), 1-16.

Goldring, M. B., Otero, M., Plumb, D. A., Dragomir, C., Favero, M., El Hachem, K., \& Marcu, K. B. (2011). Roles of inflammatory and anabolic cytokines in cartilage metabolism: signals and multiple effectors converge upon MMP-13 regulation in osteoarthritis. European cells \& materials, 21, 202.

Goldring, S. R. (2009). Role of bone in osteoarthritis pathogenesis. Medical Clinics of North America, 93(1), 25-35.

Goldring, S. R., \& Goldring, M. B. (2016). Changes in the osteochondral unit during osteoarthritis: structure, function and cartilage-bone crosstalk. Nature Reviews Rheumatology, 12(11), 632.

Hegde, V., Shonuga, O., Ellis, S., Fragomen, A., Kennedy, J., Kudryashov, V., \& Lane, J. M. (2014). A prospective comparison of 3 approved systems for autologous bone marrow concentration demonstrated nonequivalency in progenitor cell number and concentration. Journal of orthopaedic trauma, 28(10), 591-598.

Hernigou, P., Homma, Y., Lachaniette, C. H. F., Poignard, A., Allain, J., Chevallier, N., \& Rouard, H. (2013). Benefits of small volume and small syringe for bone marrow aspirations of mesenchymal stem cells. International orthopaedics, 37(11), 2279-2287.

Hong, S. J., Traktuev, D. O., \& March, K. L. (2010). Therapeutic potential of adipose-derived stem cells in vascular growth and tissue repair. Current opinion in organ transplantation, 15(1), 86-91.

Jamshidi, A., Pelletier, J. P., \& Martel-Pelletier, J. (2019). Machine-learning-based patient-specific prediction models for knee osteoarthritis. Nature Reviews Rheumatology, 15(1), 49-60

Jäger, M., Hernigou, P., Zilkens, C., Herten, M., Li, X., Fischer, J., \& Krauspe, R. (2010). Cell therapy in bone healing disorders. Orthopedic reviews, 2(2).

Kim, G. B., Seo, M. S., Park, W. T., \& Lee, G. W. (2020). Bone marrow aspirate concentrate: Its uses in osteoarthritis. International journal of molecular sciences, 21(9), 3224

Kim, J. D., Lee, G. W., Jung, G. H., Kim, C. K., Kim, T., Park, J. H., \& You, Y. B. (2014). Clinical outcome of autologous bone marrow aspirates concentrate (BMAC) injection in degenerative arthritis of the knee. European Journal of Orthopaedic Surgery \& Traumatology, 24(8), 1505-1511.

Kim, J. R., Yoo, J. J., \& Kim, H. A. (2018). Therapeutics in osteoarthritis based on an understanding of its molecular pathog enesis. International journal of molecular sciences, 19(3), 674 .

Krishnasamy, P., Hall, M., \& Robbins, S. R. (2018). The role of skeletal muscle in the pathophysiology and management of knee osteoarthritis. Rheumatology, 57(suppl_4), iv22-iv33.

Lawrence, R. C., Felson, D. T., Helmick, C. G., Arnold, L. M., Choi, H., Deyo, R. A., \& National Arthritis Data Workgroup. (2008). Estimates of the prevalence of arthritis and other rheumatic conditions in the United States: Part II. Arthritis \& Rheumatism, 58(1), 26-35.

Lespasio, M. J., Piuzzi, N. S., Husni, M. E., Muschler, G. F., Guarino, A. J., \& Mont, M. A. (2017). Knee osteoarthritis: a primer. The Permanente Journal, 21.

Loeser, R. F., Goldring, S. R., Scanzello, C. R., \& Goldring, M. B. (2012). Osteoarthritis: a disease of the joint as an organ. Arthritis and rheumatism, 64(6), 1697. 
Mabey, T., Honsawek, S., Tanavalee, A., Yuktanandana, P., Wilairatana, V., \& Poovorawan, Y. (2016). Plasma and synovial fluid inflammatory cytokine profiles in primary knee osteoarthritis. Biomarkers, 21(7), 639-644.

Mancuso, P., Raman, S., Glynn, A., Barry, F., \& Murphy, J. M. (2019). Mesenchymal stem cell therapy for osteoarthritis: the critical role of the cell secretome. Frontiers in bioengineering and biotechnology, 7, 9.

Musumeci, G. (2016). The effect of mechanical loading on articular cartilage.

Pierini, M., Di Bella, C., Dozza, B., Frisoni, T., Martella, E., Bellotti, C., \& Donati, D. (2013). The posterior iliac crest outperforms the anterior iliac crest when obtaining mesenchymal stem cells from bone marrow. JBJS, 95(12), 1101-1107.

Pittenger, M. F., Discher, D. E., Péault, B. M., Phinney, D. G., Hare, J. M., \& Caplan, A. I. (2019). Mesenchymal stem cell perspective: cell biology to clinical progress. NPJ Regenerative medicine, 4(1), 1-15.

Piuzzi, N. S., Khlopas, A., Newman, J. M., Ng, M., Roche, M., Husni, M. E., \& Muschler, G. (2018). Bone marrow cellular therapies: novel therapy for knee osteoarthritis. The journal of knee surgery, 31(01), 022-026.

Prodromos, C., Finkle, S., Rumschlag, T., \& Lotus, J. (2020). Autologous Mesenchymal Stem Cell Treatment is Consistently Effective for the Treatment of Knee Osteoarthritis: The Results of a Systematic Review of Treatment and Comparison to a Placebo Group. Medicines, $7(8), 42$.

Ren, G., Zhang, L., Zhao, X., Xu, G., Zhang, Y., Roberts, A. I., \& Shi, Y. (2008). Mesenchymal stem cell-mediated immunosuppression occurs via concerted action of chemokines and nitric oxide. Cell stem cell, 2(2), 141-150.

Rezende, M. U. D., Silva, R. B. B. D., Bassit, A. C. F., Tatsui, N. H., Sadigursky, D., \& Bolliger Neto, R. (2011). Efeito do plasma rico em plaquetas na apoptose pós-traumática de condrócitos. Acta Ortopédica Brasileira, 19(2), 102-105.

Roos, E. M., \& Arden, N. K. (2016). Strategies for the prevention of knee osteoarthritis. Nature Reviews Rheumatology, $12(2), 92$.

Sanchez, C., Pesesse, L., Gabay, O., Delcour, J. P., Msika, P., Baudouin, C., \& Henrotin, Y. E. (2012). Regulation of subchondral bone osteoblast metabolism by cyclic compression. Arthritis \& Rheumatism, 64(4), 1193-1203.

Sellam, J., \& Berenbaum, F. (2010). The role of synovitis in pathophysiology and clinical symptoms of osteoarthritis. Nature Reviews Rheumatology, 6(11), 625.

Shapiro, S. A., Arthurs, J. R., Heckman, M. G., Bestic, J. M., Kazmerchak, S. E., Diehl, N. N., \& O’Connor, M. I. (2019). Quantitative T2 MRI mapping and 12-month follow-up in a randomized, blinded, placebo controlled trial of bone marrow aspiration and concentration for osteoarthritis of the knees. Cartilage, 10(4), 432-443.

Shapiro, S. A., Kazmerchak, S. E., Heckman, M. G., Zubair, A. C., \& O’Connor, M. I. (2017). A prospective, single-blind, placebo-controlled trial of bone marrow aspirate concentrate for knee osteoarthritis. The American journal of sports medicine, 45(1), 82-90.

Shetty, A. A., Kim, S. J., Ahmed, S., Trattnig, S., Kim, S. A., \& Jang, H. J. (2018). A cost-effective cell-and matrix-based minimally invasive single-stage chondroregenerative technique developed with validated vertical translation methodology. The Annals of The Royal College of Surgeons of England, 100(3), 240-246.

Silverwood, V., Blagojevic-Bucknall, M., Jinks, C., Jordan, J. L., Protheroe, J., \& Jordan, K. P. (2015). Current evidence on risk factors for knee osteoarthritis in older adults: a systematic review and meta-analysis. Osteoarthritis and cartilage, 23(4), 507-515.

Wu, C. L., Harasymowicz, N. S., Klimak, M. A., Collins, K. H., \& Guilak, F. (2020). The role of macrophages in osteoarthritis and cartilage repair. Osteoarthritis and cartilage.

Xia, B., Chen, D., Zhang, J., Hu, S., Jin, H., \& Tong, P. (2014). Osteoarthritis pathogenesis: a review of molecular mechanisms. Calcified tissue international, 95(6), 495-505.

Yang, Q., Zhou, Y., Cai, P., Fu, W., Wang, J., Wei, Q., \& Li, X. (2019). Up-regulated HIF-2 $\alpha$ contributes to the Osteoarthritis development through mediating the primary cilia loss. International immunopharmacology, 75, 105762.

Zhang, W., Moskowitz, R. W., Nuki, G., Abramson, S., Altman, R. D., Arden, N., \& Tugwell, P. (2007). OARSI recommendations for the management of hip and knee osteoarthritis, part I: critical appraisal of existing treatment guidelines and systematic review of current research evidence. Osteoarthritis and cartilage, 15(9), 981-1000.

Zhang, W., Moskowitz, R. W., Nuki, G., Abramson, S., Altman, R. D., Arden, N., \& Tugwell, P. (2008). OARSI recommendations for the management of hip and knee osteoarthritis, Part II: OARSI evidence-based, expert consensus guidelines. Osteoarthritis and cartilage, 16(2), 137-162.

Zhang, W., Nuki, G., Moskowitz, R. W., Abramson, S., Altman, R. D., Arden, N. K., \& Tugwell, P. (2010). OARSI recommendations for the management of hip and knee osteoarthritis: part III: Changes in evidence following systematic cumulative update of research published through January 2009. Osteoarthritis and cartilage, 18(4), 476-499. 